\title{
Protectin Conjugates in Tissue Regeneration 1 Restores Lipopolysaccharide-Induced Pulmonary Endothelial Glycocalyx Loss via ALX/SIRT1/NF- kappa B Axis
}

\section{Xin-Yang Wang}

Wenzhou Medical University Second Affiliated Hospital

Xin-Yu Li

Wenzhou Medical University Second Affiliated Hospital

Cheng-Hua Wu

Wenzhou Medical University Second Affiliated Hospital

Yu Hao

Wenzhou Medical University Second Affiliated Hospital

Pan-Han Fu

Wenzhou Medical University Second Affiliated Hospital

Hong-Xia Mei

Wenzhou Medical University Second Affiliated Hospital

\section{Fang Chen}

Wenzhou Medical University Second Affiliated Hospital

Yu-Qiang Gong

Wenzhou Medical University Second Affiliated Hospital

Sheng-Wei Jin

Wenzhou Medical University Second Affiliated Hospital

Hui Li ( $\nabla$ judy198406@sina.com )

wenzhou medical university https://orcid.org/0000-0003-4212-9155

\section{Research}

Keywords: PCTR1, glycocalyx, HPA, EXT-1, SIRT1

Posted Date: November 11th, 2020

DOl: https://doi.org/10.21203/rs.3.rs-102371/v1

License: (c) (1) This work is licensed under a Creative Commons Attribution 4.0 International License. Read Full License 
Version of Record: A version of this preprint was published at Respiratory Research on July 3rd, 2021. See the published version at https://doi.org/10.1186/s12931-021-01793-x. 


\section{Abstract}

Background: Endothelial glycocalyx loss is integral to increased pulmonary vascular permeability in sepsis-related acute lung injury. Protectin conjugates in tissue regeneration 1 (PCTR1) is a novel macrophage-derived lipid mediator exhibiting potential anti-inflammatory and pro-resolving benefits.

Methods: PCTR1 was administrated intraperitoneally with $100 \mathrm{ng} / \mathrm{mouse}$ after lipopolysaccharide (LPS) challenged. Survival rate and lung function were used to evaluate the protective effects of PCTR1. Lung inflammation response was observed by morphology and inflammatory cytokines level. Endothelial glycocalyx and its related key enzymes were measured by immunofluorescence, ELISA, and Western blot. Afterward, related-pathways inhibitors were used to identify the mechanism of endothelial glycocalyx response to PCTR1 in mice and human umbilical vein endothelial cells (HUVECs) after LPS administration.

Results: In vivo, we show that PCTR1 protects mice against lipopolysaccharide (LPS)-induced sepsis, as shown by enhanced the survival and pulmonary function, decreased the inflammatory response in lungs and peripheral levels of inflammatory cytokines such as tumor necrosis factor-a, interleukin-6, and interleukin-1 $\beta$. Moreover, PCTR1 restored lung vascular glycocalyx and reduced serum heparin sulphate (HS), syndecan-1 (SDC-1), and hyaluronic acid (HA) levels. Furthermore, we found that PCTR1 downregulated heparanase (HPA) expression to inhibit glycocalyx degradation and upregulated exostosin-1 (EXT-1) protein expression to promote glycocalyx reconstitution. Besides, we observed that BAY11-7082 blocked glycocalyx loss induced by LPS in vivo and in vitro, and BOC-2区ALX antagonist $\$ or EX527『SIRT1 inhibitor》 abolished the restoration of HS in response to PCTR1.

Conclusion: PCTR1 protects endothelial glycocalyx via ALX receptor by regulating SIRT1/NF-KB pathway, suggesting PCTR1 may be a significant therapeutic target for sepsis-related acute lung injury.

\section{Background}

Sepsis is a common condition responsible for high morbidity and mortality worldwide ${ }^{[1]}$. Acute respiratory distress syndrome (ARDS), a severe sepsis complication, contributes to its high mortality ${ }^{[2,3]}$. Currently, there are no treatments or prevention available for sepsis-related lung injury. The endothelium regulates the pathophysiology of lung injury ${ }^{[4]}$. The endothelial glycocalyx (EGL) primarily consists of glycosaminoglycans and proteoglycans,coating all healthy vascular endothelium ${ }^{[5]}$. Shedding of the endothelial glycocalyx is mainly driven by activation of heparan sulfate-specific heparanase, HPA ${ }^{[6]}$. Activated HPA promotes the degradation of the glycosaminoglycans and proteoglycans, and increases the level of glycocalyx components in the bloodstream, mainly including heparin sulphate (HS), hyaluronic acid (HA), and syndecan-1 (SDC-1) ${ }^{[6]}$. Exostosin-1 (EXT-1), a heparan sulfate elongation gene, is involved in glycocalyx synthesis and restitution, which is delayed in sepsis ${ }^{[8]}$. Damaged glycocalyx leads to an increase in permeability to proteins and fluids, causing interstitial leakage ${ }^{[9]}$. Therefore, it is critical to improve glycocalyx reconstitution for the treatment of sepsis. 
Protectin conjugates in tissue regeneration 1 (PCTR1) is a newly discovered member of the specialized pro-resolving mediators (SPMs) from docosahexaenoic acid (DHA) belonging to the peptide-conjugated Protectins ${ }^{[10,11]}$. PCTR1 is identified and present in vivo before infection, and increases after LPS-induced inflammation, especially in inflammation resolution phrase ${ }^{[12]}$. Notably, PCTR1 not only enhances inflammation resolution by promoting macrophage efferocytosis and phagocytosis in infectious disease models but also improves tissue reparation ${ }^{[13,14]}$. However, the effect of PCTR1 on endothelial glycocalyx is still undefined.

Herein, the effects of PCTR1 on LPS-induced inflammation were investigated using a murine model for sepsis. Specifically, we examined its impact on survival rate, lung function, and inflammatory injury in the lungs. Next, we investigated its effect on lung vascular endothelial glycocalyx. Finally, we determined the effect of the receptor, as well as pathways targeted by PCTR1 on LPS-induced glycocalyx loss to gain insights into the underlying mechanisms.

\section{Methods}

\section{Animal preparation}

Specific pathogen-free (SPF) adult male C57BL/6 mice (6-8 wk) were bought from SLAC Laboratory Animal CO. (Shanghai, China). Before the experiments, the mice were kept in standard cages in a house controlled to a $12 \mathrm{hr}$ light/dark cycle with temperature maintained at $22-24{ }^{\circ} \mathrm{C}$, humidity at $50-60 \%$ ) and SPF environment in Wenzhou Medical University. The mice were allowed free access to water and food. All animal procedures conformed to the Guide for the Care and Use of Laboratory Animals. This was approved by the Animal Studies Ethics Committee of Wenzhou Medical University.

\section{Animal experimental groups}

Mice were intraperitoneally injected with LPS (15 mg/kg, serotype 055: B5; Sigma, Saint Louis, MO, USA) for survival experiments and LPS (10 mg/kg) for other experiments. The effect of PCTR1 on LPS-induced sepsis model was determined by establishing four experimental groups: control group, LPS group, LPS+PCTR1 group, PCTR1 group. For the PCTR1 and control groups, the mice were intraperitoneally injected with PCTR1 100ng/mouse (Cayman Chemical, Ann Arbor, MI, USA) or an equal saline volume. Next, to determine the mechanism of PCTR1, four groups were established: control group, LPS group, LPS + PCTR1 group, LPS + PCTR1 + BOC-2 group, LPS + PCTR1 + EX527 group, and LPS + BAY11-7082

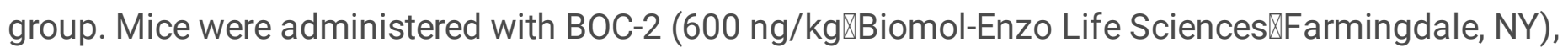
EX527 (10 mg/kg, MEC, Shanghai, China), and BAY11-7082 (20 mg/kg, Selleck, Houston, TX, USA) intraperitoneally following LPS administration. The mice were anesthetized with $1 \%$ pentobarbital and sacrificed 6hours later. We obtained blood samples via the ophthalmic artery from mice that survived in each group, and obtained lung specimens.

\section{Cell culture and experimental groups}


Human umbilical vein endothelial cells (HUVECs) were purchased from SGST (China). The cells were suspended in a DMEM medium containing $10 \%$ FBS and cultured in $25 \mathrm{~cm}^{2}$ flasks placed in an incubator controlled at $5 \% \mathrm{CO}_{2}$ at $37^{\circ} \mathrm{C}$. In all experiments, HUVECs were added to the wells of six-well plates at equal concentrations and were further sub-divided into six groups: control group, LPS group, LPS + PCTR1 group, LPS + PCTR1 + DMSO group (DMSO was used as the solvent for EX527), LPS + PCTR1 + EX527 group and LPS + BAY11-7082 group. DMSO, EX527 $(1 \mu \mathrm{M})$, and BAY11-7082 $(1 \mu \mathrm{M})$ were added to cells for 24 hours followed by LPS $(1 \mu \mathrm{g} / \mathrm{ml})$ and/or PCTR1 $(100 \mathrm{nM})$ stimulation.

\section{Invasive evaluation of respiratory mechanics}

The lung function assay was conducted using a flexiVent system (Scireq, Montreal, QC, Canada), as described previously ${ }^{[15]}$. In brief, mice were anesthetized with $90 \mathrm{mg} / \mathrm{kg}$ pentobarbital sodium injected intraperitoneally and then tracheotomized. Vecuronium bromide was administered into each mice via intravenous injection, followed by mechanical ventilation using a computer-moderated small-animal ventilator. A model of deep inflation was used to record the inspiratory capacity (IC). Cst (quasistatic compliance) was assessed based on the PV curves.

\section{Lung Morphology}

Left lungs were excised after anesthetization, and fixed overnight with $4 \%$ paraformaldehyde at room temperature. This was followed by staining of $5-\mu \mathrm{m}$ sections using hematoxylin and eosin (HE) and microscopic examination. The lung injury score was determined based on the level of infiltration of inflammatory cells, hyperemia, and the thickness of the alveolar wall.

\section{Lung vascular permeability assay}

The evans blue dye (EBD) extravasation was utilized to assess pulmonary vascular permeability. Five and a half hours following LPS administration, EBD $(20 \mathrm{mg} / \mathrm{kg})$ was injected through the caudal vein. After the circulation of the dye for 30 minutes, perfusion of lungs was performed with saline $(25 \mathrm{ml})$ under anaesthesia. Subsequently, the lungs were excised, blotted dry, weighed, and homogenized in formamide. After overnight extraction, the tissue fluid was centrifugated for 10 minutes at $12,000 \times \mathrm{g}$. A microplate reader was used to read the EBD concentration of the resultant supernatant at $620 \mathrm{~nm}$ absorbance.

\section{Wet-to-dry lung weight ratio}

The index of pulmonary edema was determined from the wet-to-dry (W/D) lung weight ratio. Portions of the harvested wet left lungs were weighed and heated in an oven at $60^{\circ} \mathrm{C}$ for 48 hours. The W/D ratio was then determined after re-weighing the portions as dry weight.

\section{ELISA}

Blood samples were obtained to determine level serum inflammatory cytokines, as well as the degradation products of glycocalyx in circulation. The blood was collected using the orbital sinus 
extraction method under anaesthesia. The serum was isolated from the blood samples for ELISA tests. Serum levels of cytokines including TNF-a, IL-1 $\beta$, and IL- 6 and glycocalyx-related proteins including HS, SDC-1, and HA were quantified using R\&D systems and Boyun biotech ELISA kits following protocols provided by the manufacturer.

\section{Western blot}

Lung tissues were homogenized with lysis buffer (RIPA: PMSF $=1: 1)$ to obtain protein samples. The tissue homogenates were ultrasonicated and then centrifuged for $30 \mathrm{~min}$ at $12,000 \times \mathrm{g}$. The concentration of proteins in the supernatants was measured using the BCA kit. An equal amount of protein from each group was loaded onto 10\% SDS-PAGE gels and resolved. The proteins were electro-transferred onto PVDF membranes. After being blocked with $10 \%$ milk for 2 hours, membranes were incubated with the primary antibodies: EXT-1 (1:2000, GeneTex, Irvine, CA, USA), HPA (1:1000, Abcam, Cambridge, MA, USA), p65 (1:1000, Abcam, Cambridge, MA, USA), p-p65 (1:1000, Abcam, Cambridge, MA, US), SIRT1 (1:1000, Abcam, Cambridge, MA, USA), and $\beta$-actin (1:1000, BOYUN, Shanghai, China) overnight at $4{ }^{\circ} \mathrm{C}$. Next, the membranes were washed thrice and incubated with secondary antibody (1:3000, BOYUN, Shanghai, China) at room temperature for 1 hour followed by another three-times wash. The protein bands were analyzed by Image Quant LAS 4000 mini (GE Healthcare Bio-Sciences AB, Uppsala, Sweden). The band intensity was analyzed with ImageJ.

\section{Immunofluorescence}

Immunofluorescence was carried out using lung tissues and HUVECs. Lung sections were prepared for immunofluorescence after deparaffinized, dehydrated and antigen retrieval. This was followed by fixation of the HUVECs in $4 \%$ paraformaldehyde. Subsequently, the fixed cells and tissue samples were mounted on the cover glass, blocked with donkey serum (Solarbio, Beijing, China) and probed with HSPG2antibody (1:200). After rinsing thrice with PBS, the fixed cells and tissue sections were incubated with the secondary antibody $(1: 200)$ at $37^{\circ} \mathrm{C}$ for 1 hour and further incubated with DAPI (Abcam) for 5 min. Eventually, both fixed cells and sectioned tissues were covered with an antifade mounting medium (Solarbio, Beijing, China) for fluorescence microscopy (Leica).

\section{Data analysis}

The mean values are presented as the mean \pm SD and were analyzed with GraphPad Prism 7.0 software. Mean values of groups were compared one-way ANOVA followed by Tukey's post hoc test for multiple comparisons. Kaplan-Meier analysis was conducted to evaluate survival and a log-rank (Mantel-Cox) test was employed to determine statistical significance. $P<.05$ was considered statistically significant.

\section{Results}

PCTR1 improves survival and lung functionin mice after LPS administration 
We first determine the effect of PCTR1 on septic mice by survival curve analysis. PCTR1 significantly promoted survival in mice subjected to LPS (15 mg/kg) injected (Fig. 1a). Next, we performed a lung function test on LPS (10 mg/kg)-induced endotoxemia mice. Compared with the saline group, mice with LPS challenge showed a statistically significant reduction in IC and Cst, whereas PCTR1 enhanced IC and Cst significantly (Fig. 1b, c).

\section{PCTR1 alleviates LPS-stimulated lung inflammatory response in mice}

The histology in the control group showed normal lung conditions in mice. Notably, lung tissues in the LPS group were remarkably damaged, involving alveolar disarray, as well as infiltration level of inflammatory cells as indicated by increases in lung injury scores unlike mice in the control group. There was no marked change in morphologic features in the LPS + PCTR1 group. There was no remarkable difference between the control and the PCTR1 group (Fig. 2a, b). Accordingly, the peripheral levels of inflammatory cytokines, e.g., TNF-a, IL-1 $\beta$, and IL- 6 were remarkably upregulated in the LPS group relative to the control group and were lower in the PCTR1 treatment group compared with the LPS group (Fig. 2ce). Furthermore, to determine pulmonary vascular permeability in mice, the EBD test and W/D ratio of lung tissues were assessed. As expected, PCTR1 significantly inhibited LPS induced EBD and W/D increase of lung tissues (Fig. 2f, g).

\section{PCTR1 restores LPS-induced endothelial glycocalyx damage in mice}

On account of the importance of endothelial glycocalyx in maintaining vascular permeability, we tested the effect of PCTR1 on LPS-induced endothelial glycocalyx damage. Our results demonstrated that PCTR1increased the expression of HS and SDC-1 in lungs after LPS challenge (Fig. 3a-d). Accordingly, PCTR1 reduces the peripheral concentration of HS, SDC-1 and HA in septic mice (Fig. 3e-g).

\section{PCTR1 regulates HPA and EXT-1 expression in LPS-triggered sepsis in mice}

The loss of endothelial glycocalyx induced pulmonary vascular hyperpermeability. HPA and EXT-1 are key enzymes involving in maintaining endothelial glycocalyx integrity via regulating degradation and reconstitution, respectively. PCTR1 decreased HPA expression and increased EXT-1 expression in LPSinduced septic mice. In line with a previous study, the lower SIRT1 expression and its downstream, the higher NF-KB p65 phosphorylation ( $p$-p65) expression was found in LPS group mice; by contrast, PCTR1 inhibited those changes in mice after LPS challenge (Fig. 4a, b). Together with the finding that, we postulate that PCTR1 might restores HPA and EXT-1 mediated endothelial glycocalyx dynamic balance through enhancing SIRT1 expression. As shown in our results, the inhibition of NF-KB blocked the effect of LPS on HPA and EXT-1, whilst the inhibition of SIRT1 blocked the effect of PCTR1 on HPA and EXT-1. Furthermore, we found that the effect of PCTR1 was abolished in the presence of BOC-2, the GFPR inhibitor (Fig. 4c, d).

\section{PCTR1 protects the endothelial glycocalyx bymodulating SIRT1/NF-KB pathways via ALX receptor}


To determine whether PCTR1 restores endothelial glycocalyx via ALX/SIRT1/NF-KB, we assessed the HS expression in lung sections of each group by immunofluorescence. As expected, the effect of LPS on HS was inhibited in the presence of BAY11-7082, and the effect of PCTR1 on HS was abolished in the presence of BOC-2 or EX527 (Fig. 5a, b). Consistent with the experiment results in vivo, we found that BAY11-7082 inhibited LPS-induced HS loss, and EX527 inhibited PCTR1-induced HS restoration in HUVECs after LPS stimulation (Fig. 5c, d).

\section{Discussion}

This study results provided research evidence of the protective effects of PCTR1 in a murine model of LPS injection. We first observed that PCTR1 inhibited the infiltration of neutrophils, the generation of TNFa,IL-1 $\beta$, as well as IL- 6 and LPS-provoked hyperpermeability in the lungs, with the outcome of enhanced survival and lung function. Next, we demonstrated that PCTR1 reduced lung endothelial glycocalyx degradation and improved glycocalyx reconstitution after LPS administration. Furthermore, we found PCTR1 decreased glycocalyx degradation factor HPA and increased glycocalyx key synthetase EXT-1 via ALX/SIRT1/NF-KB, restoring endothelial glycocalyx consequently (Fig. 6).

PCTR1 is a new endogenous mediator with the potential to promote inflammation resolution via $G$ protein-coupled receptors(ALX) ${ }^{[16]}$. It was demonstrated that PCTR1 is increased to enhance macrophage recruitment and phagocytosis during the inflammation resolution phase ${ }^{[12]}$. Moreover, PCTR1 generation in peritoneal lavage is decreased in vagotomized mice, suggesting that PCTR1 is controlled by local vagal to improve inflammation resolution ${ }^{[13]}$. Our previous studies demonstrated that PCTR1 reduced LPS-induced inflammation in mice via regulating linoleic acid metabolism, and improved pulmonary edema fluid clearance by activating the sodium channel and lymphatic drainage in LPS-triggered rat model ${ }^{[17,18]}$. Similarly, we also observed that PCTR1 improved lung function and lung vascular leakage after LPS challenge in mice.

Increasing evidence demonstrates that the intact glycocalyx contributes to the maintenance of vascular integrity and leukocyte trafficking ${ }^{[19]}$. Whilst, after an acute injury, glycocalyx is degraded by HPA, and the level of glycocalyx components such as HS, SDC-1 and HA is elevated in the bloodstream, which could predict outcomes of patients with sepsis-induced organ dysfunction, including ARDS ${ }^{[20,21]}$. In sepsis, EXT1 expression is decreased, and glycocalyx reconstitution is delayed, which increases sepsis severity ${ }^{[22]}$. Previous studies has reported that enhancing endothelial fibroblast growth factor receptor 1 signaling improves EXT-1-mediated pulmonary glycocalyx reconstitution in sepsis ${ }^{[8,23]}$. Thus, endothelial glycocalyx protection has been considered as a therapeutic strategy to treat sepsis. Herein, we found that PCTR1 unregulated EXT-1 expression and downregulated HPA expression, promoting glycocalyx reconstitution in sepsis model via ALX receptor consequently.

It has been demonstrated that SIRT1 is a negative regulator of inflammation ${ }^{[24]}$. Previous studies reported that endothelial glycocalyx decreased in vivo and in vitro with a defect in SIRT1 deacetylase activity ${ }^{[25,26]}$. 
Consistently, we found that the protective effect of PCTR1 on glycocalyx was abolished in the presence of the inhibitor of SIRT1. SIRT1 has been reported to suppress inflammatory responses by inhibiting NFKB signaling ${ }^{[27]}$. SIRT1 not only stimulates oxidative energy production but also inhibits transcription by deacetylating $p 65$ and suppresses inflammation ${ }^{[28,29]}$. On the other hand, the increases of HPA expression in various inflammation and cancer models with the increase of NF-kB P65 phosphorylation ${ }^{[30]}$. It is reported that NF-KB inhibitor attenuated HPA expression ${ }^{[30,31]}$. In our study, we found that LPS increased HPA expression and decreased EXT-1 expression via activating NF-KB, degrading glycocalyx consequently in vivo and in vitro. Whilst, PCTR1 induced SIRT1 expression and reduced NF-KB phosphorylation after LPS administration and reversed the effect of LPS on glycocalyx.

\section{Conclusions}

This study indicates that PCTR1 reduces endothelial glycocalyx loss and improves glycocalyx reconstitution by modulating glycocalyx key enzymes including HPA and EXT-1 through ALX/SIRT1/NFKB cascade after LPS treatment. The preservation of the glycocalyx attenuates lung inflammation response and vascular endothelial hyperpermeability, and further restores lung function and survival rate after LPS administration in mice. This study not only identifies a protective action of PCTR1 in LPSinduced sepsis mice, but the results may also open a new therapeutic strategy with PCTR1 for other vascular-related diseases.

\section{Abbreviations}

PCTR1囚Protectin conjugates in tissue regeneration 1; LPS: lipopolysaccharide; HS: heparin sulphate; SDC1: syndecan-1; HA: hyaluronic acid; HPA: heparanase; EXT-1: exostosin-1; ARDS: acute respiratory distress syndrome; EGL: endothelial glycocalyx; SPMs: specialized pro-resolving mediators; DHA:

docosahexaenoic acid; SPF: specific pathogen-free; HUVECs: human umbilical vein endothelial cells; IC: inspiratory capacity; Cst: quasistatic compliance; HE: hematoxylin and eosin; EBD: evans blue dye; W/D: wet-to-dry; GPCR: G protein-coupled receptors; SIRT1: sirtuin 1

\section{Declarations}

\section{Ethics approval and consent to participate}

All animal experiments in this study were performed in accordance with the Guide for the Care and Use of Laboratory Animals of Wenzhou Medical University.

\section{Consent for publication}

Not applicable.

\section{Availability of data and materials}


All supporting data are available from the corresponding author upon reasonable request.

\section{Competing interests}

There are no conflict of interest to declare.

\section{Funding}

The study was supported by funds from the Natural Science Foundation of Zhejiang Province (No. LQ20H150003, No. LQ19H150001, No. LY19H150002), Zhejiang Science and Technology department key research and development program (No. 2019C03011), Wenzhou Science and Technology Bureau Project (No. Y20190087, No. Y20190118).

\section{Authors' contributions}

Xin-Yang Wang: Investigation. Xin-Yu Li: Investigation. Chen-Hua Wu: Investigation. Yu Hao:

Methodology, Software. Pan-Han Fu: Methodology, Software. Hong-Xia Mei: Investigation. Fang Chen: Investigation. Yu-Qiang Gong: Conceptualization. Sheng-Wei Jin: Conceptualization, Funding acquisition, Validation. Hui Li: Conceptualization, Writing, Validation, Funding acquisition.

\section{Acknowledgements}

We thank Xiao-Ying Huang for lung function test. We thank all mice in our experiment for helping us to identify the hypothesis.

\section{References}

1. Cecconi M, Evans L, Levy M, Rhodes A: Sepsis and septic shock. Lancet 2018, 392:75-87.

2. Leaf DE, Waikar SS: Rosuvastatin for sepsis-associated ARDS. N Engl J Med 2014, 371:968.

3. Englert JA, Bobba C, Baron RM: Integrating molecular pathogenesis and clinical translation in sepsisinduced acute respiratory distress syndrome. JCI Insight 2019, 4.

4. Huber-Lang M, Lambris JD, Ward PA: Innate immune responses to trauma. Nat Immuno/2018, 19:327-341.

5. Yang Y, Schmidt EP: The endothelial glycocalyx: an important regulator of the pulmonary vascular barrier. Tissue Barriers 2013, 1.

6. Chappell D, Jacob M, Rehm M, Stoeckelhuber M, Welsch U, Conzen P, Becker BF: Heparinase selectively sheds heparan sulphate from the endothelial glycocalyx. Biol Chem 2008, 389:79-82.

7. Schmidt EP, Yang Y, Janssen WJ, Gandjeva A, Perez MJ, Barthel L, Zemans RL, Bowman JC, Koyanagi DE, Yunt ZX, et al: The pulmonary endothelial glycocalyx regulates neutrophil adhesion and lung injury during experimental sepsis. Nat Med 2012, 18:1217-1223.

8. Mooij HL, Cabrales P, Bernelot Moens SJ, Xu D, Udayappan SD, Tsai AG, van der Sande MA, de Groot E, Intaglietta M, Kastelein JJ, et al: Loss of function in heparan sulfate elongation genes EXT1 and 
EXT 2 results in improved nitric oxide bioavailability and endothelial function. J Am Heart Assoc 2014, 3:e001274.

9. LaRiviere WB, Schmidt EP: The Pulmonary Endothelial Glycocalyx in ARDS: A Critical Role for Heparan Sulfate. Curr Top Membr 2018, 82:33-52.

10. Hansen TV, Vik A, Serhan CN: The Protectin Family of Specialized Pro-resolving Mediators: Potent Immunoresolvents Enabling Innovative Approaches to Target Obesity and Diabetes. Front Pharmacol 2018, 9:1582.

11. Dalli J, Chiang N, Serhan CN: Identification of 14-series sulfido-conjugated mediators that promote resolution of infection and organ protection. Proc Natl Acad Sci U S A 2014, 111:E4753-4761.

12. Ramon S, Dalli J, Sanger JM, Winkler JW, Aursnes M, Tungen JE, Hansen TV, Serhan CN: The Protectin PCTR1 Is Produced by Human M2 Macrophages and Enhances Resolution of Infectious Inflammation. Am J Pathol 2016, 186:962-973.

13. Serhan CN, de la Rosa X, Jouvene C: Novel mediators and mechanisms in the resolution of infectious inflammation: evidence for vagus regulation. J Intern Med 2019, 286:240-258.

14. Dalli J, Colas RA, Arnardottir H, Serhan CN: Vagal Regulation of Group 3 Innate Lymphoid Cells and the Immunoresolvent PCTR1 Controls Infection Resolution. Immunity 2017, 46:92-105.

15. Li H, Hao Y, Zhang H, Ying W, Li D, Ge Y, Ying B, Cheng B, Lian Q, Jin S: Posttreatment with Protectin DX ameliorates bleomycin-induced pulmonary fibrosis and lung dysfunction in mice. Sci Rep 2017, 7:46754.

16. Serhan CN: Pro-resolving lipid mediators are leads for resolution physiology. Nature 2014, 510:92101.

17. Liu YJ, Li H, Tian Y, Han J, Wang XY, Li XY, Tian C, Zhang PH, Hao Y, Gao F, Jin SW: PCTR1 ameliorates lipopolysaccharide-induced acute inflammation and multiple organ damage via regulation of linoleic acid metabolism by promoting FADS1/FASDS2/ELOV2 expression and reducing PLA2 expression. Lab Invest 2020, 100:904-915.

18. Zhang PH, Han J, Cao F, Liu YJ, Tian C, Wu CH, Smith FG, Hao Y, Jin SW: PCTR1 improves pulmonary edema fluid clearance through activating the sodium channel and lymphatic drainage in lipopolysaccharide-induced ARDS. J Cell Physio/ 2020.

19. Chelazzi C, Villa G, Mancinelli P, De Gaudio AR, Adembri C: Glycocalyx and sepsis-induced alterations in vascular permeability. Crit Care 2015, 19:26.

20. Schmidt EP, Overdier KH, Sun X, Lin L, Liu X, Yang Y, Ammons LA, Hiller TD, Suflita MA, Yu Y, et al: Urinary Glycosaminoglycans Predict Outcomes in Septic Shock and Acute Respiratory Distress Syndrome. Am J Respir Crit Care Med 2016, 194:439-449.

21. Haeger SM, Yang Y, Schmidt EP: Heparan Sulfate in the Developing, Healthy, and Injured Lung. Am J Respir Cell Mol Biol 2016, 55:5-11.

22. Joffre J, Hellman J, Ince C, Ait-Oufella H: Endothelial Responses in Sepsis. Am J Respir Crit Care Med 2020, 202:361-370. 
23. Yang Y, Haeger SM, Suflita MA, Zhang F, Dailey KL, Colbert JF, Ford JA, Picon MA, Stearman RS, Lin L, et al: Fibroblast Growth Factor Signaling Mediates Pulmonary Endothelial Glycocalyx Reconstitution. Am J Respir Cell Mol Biol 2017, 56:727-737.

24. Gao R, Ma Z, Hu Y, Chen J, Shetty S, Fu J: Sirt1 restrains lung inflammasome activation in a murine model of sepsis. Am J Physiol Lung Cell Mol Physiol 2015, 308:L847-853.

25. Lipphardt M, Dihazi H, Muller GA, Goligorsky MS: Fibrogenic Secretome of Sirtuin 1-Deficient Endothelial Cells: Wnt, Notch and Glycocalyx Rheostat. Front Physiol 2018, 9:1325.

26. Fu C, Hao S, Xu X, Zhou J, Liu Z, Lu H, Wang L, Jin W, Li S: Activation of SIRT1 ameliorates LPSinduced lung injury in mice via decreasing endothelial tight junction permeability. Acta Pharmacol $\operatorname{Sin} 2019,40: 630-641$.

27. Tilstra JS, Clauson CL, Niedernhofer LJ, Robbins PD: NF-kappaB in Aging and Disease. Aging Dis 2011, 2:449-465.

28. Kauppinen A, Suuronen T, Ojala J, Kaarniranta K, Salminen A: Antagonistic crosstalk between NFkappaB and SIRT1 in the regulation of inflammation and metabolic disorders. Cell Signa/2013, 25:1939-1948.

29. Yeung F, Hoberg JE, Ramsey CS, Keller MD, Jones DR, Frye RA, Mayo MW: Modulation of NF-kappaBdependent transcription and cell survival by the SIRT1 deacetylase. EMBO J 2004, 23:2369-2380.

30. An X, Zhang L, Yao Q, Li L, Wang B, Zhang J, He M, Zhang J: The receptor for advanced glycation endproducts mediates podocyte heparanase expression through NF-kappaB signaling pathway. $\mathrm{Mol}$ Cell Endocrinol 2018, 470:14-25.

31. Ramani VC, Vlodavsky I, Ng M, Zhang Y, Barbieri P, Noseda A, Sanderson RD: Chemotherapy induces expression and release of heparanase leading to changes associated with an aggressive tumor phenotype. Matrix Biol 2016, 55:22-34.

\section{Figures}

Figure 1

A

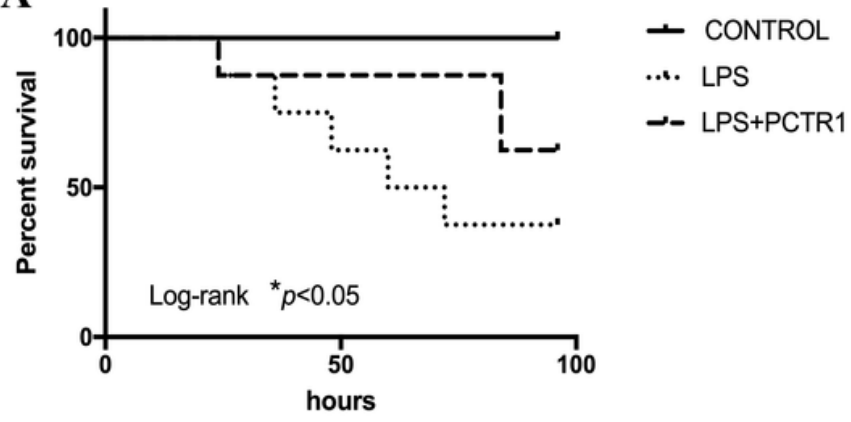

B

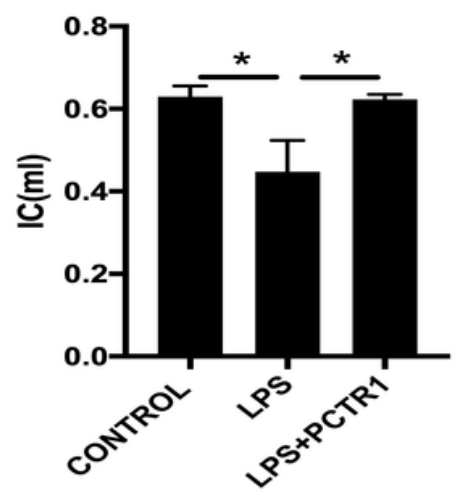

C

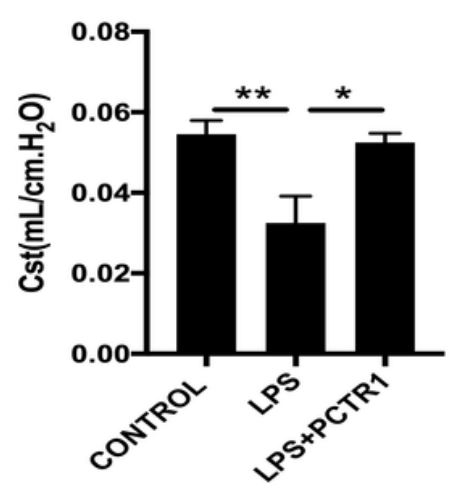


Figure 1

PCTR1 improves survival and lung function in mice after LPS administration (a) Kaplan-Meier survival curves for the LPS $(15 \mathrm{mg} / \mathrm{kg})$ with or without PCTR1 $(100 \mathrm{ng} / \mathrm{mouse})$ treated mice $(\mathrm{n}=8)$. Lung function of each group mice was measured by the flexiVent apparatus at 6 hours after LPS $(10 \mathrm{mg} / \mathrm{kg})$ with or without PCTR1 (100 ng/mouse) challenge. (b, c) IC (Inspiratory Capacity) and Cst (Static Compliance) are shown $(n=5) .{ }^{*} P<.05,{ }^{\star *} P<.01$.

\section{Figure 2}

A

CONTROL

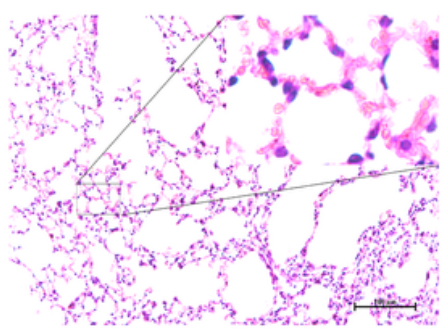

B

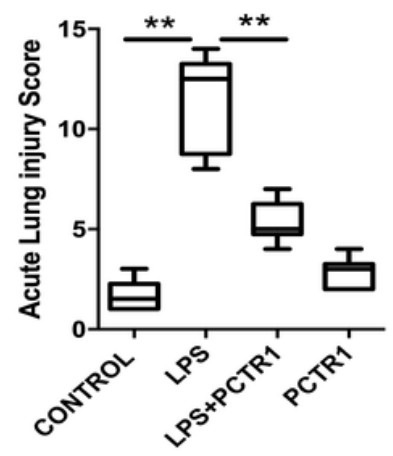

LPS

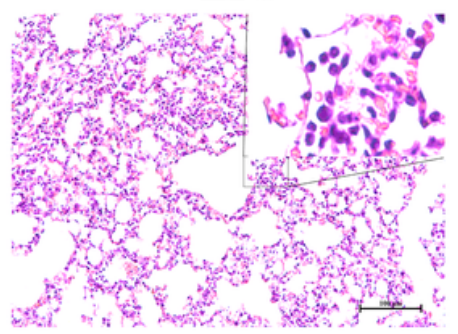

C

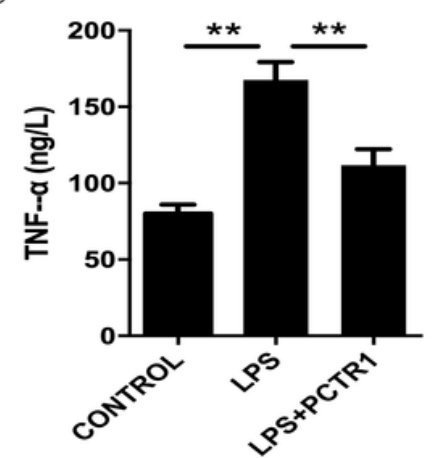

LPS+PCTR1

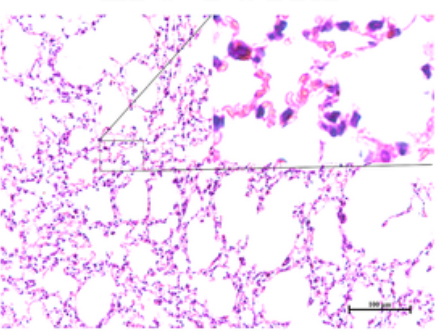

D

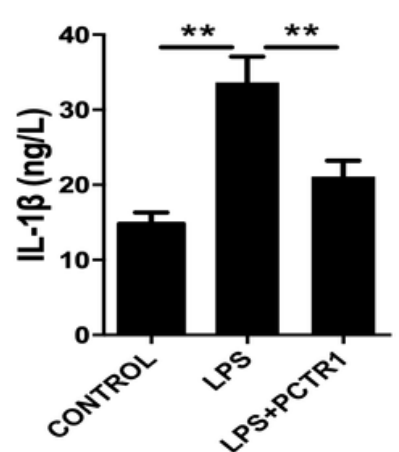

PCTR1

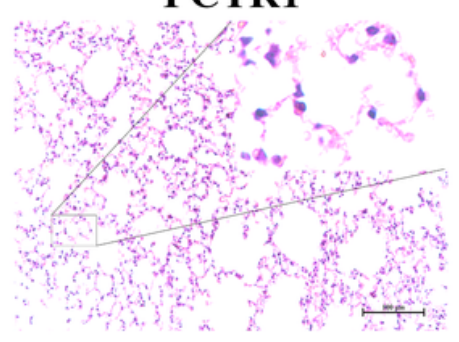

$\mathbf{E}$

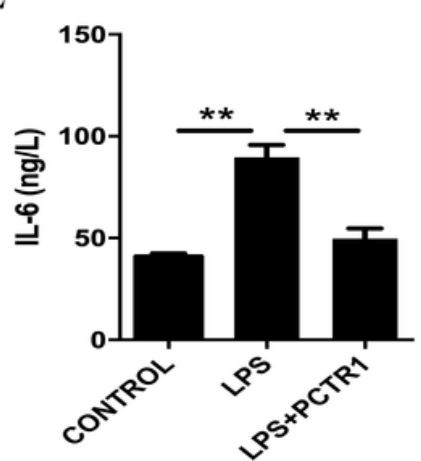

$\mathbf{F}$

G
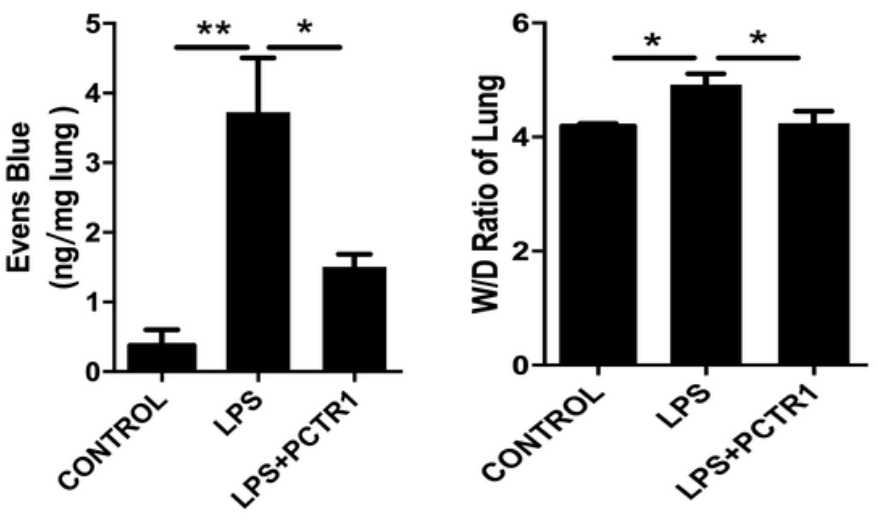

Figure 2

PCTR1 alleviates LPS-triggered lung inflammatory response in mice Mice were treated with LPS (10 $\mathrm{mg} / \mathrm{kg}$ ) with or without PCTR1 (100 ng/mouse) for 6 hours. (a) Representative images of Hematoxylin and eosin-stained in lung tissues are shown (X200). (b) Lung injury score $(n=6)$. (c-e)The serum levels of 
TNF-a, IL-1 $\beta$ and IL- 6 were quantified using ELISA $(n=5)$. ( $f, g)$ Vascular permeability of lung tissues was determined using Evans Blue Dye and W/D weight ratio ( $n=5$ each). ${ }^{*}<.05, * * P<.01$.

Figure 3

A
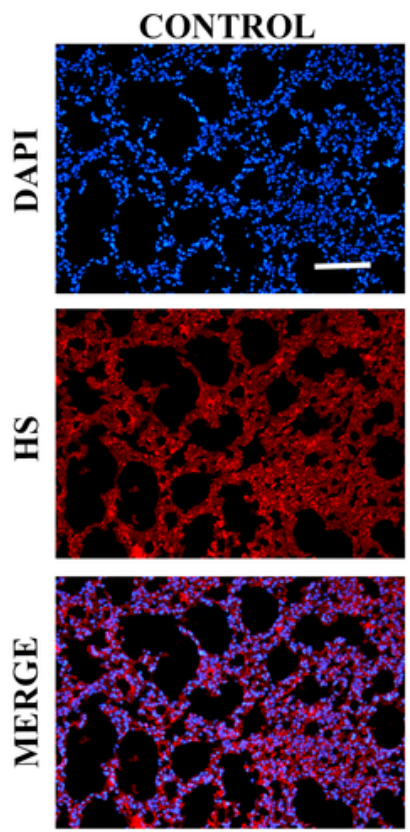

C
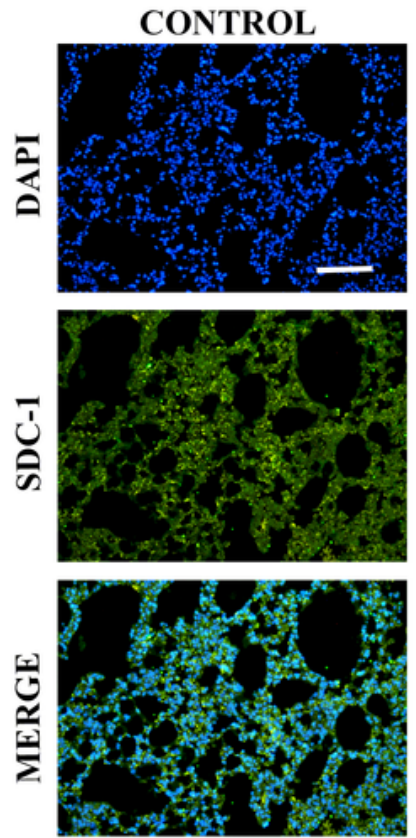

LPS
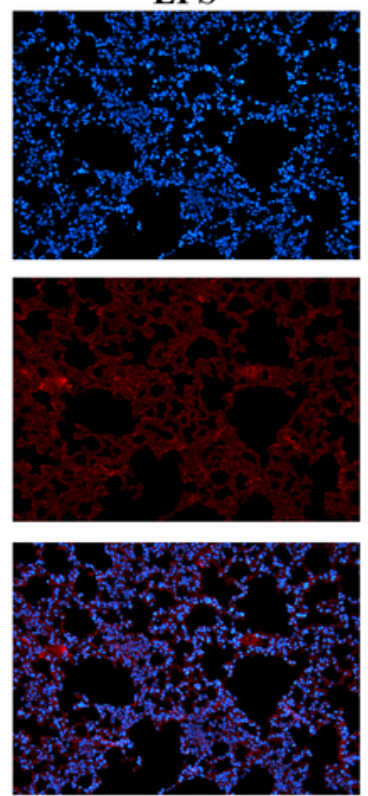

LPS
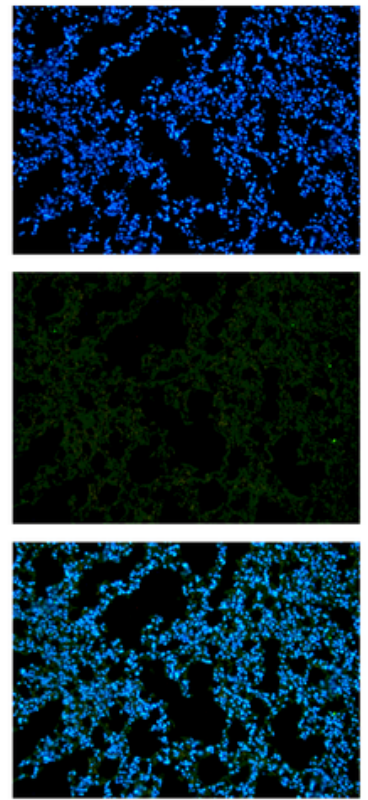

LPS+PCTR1
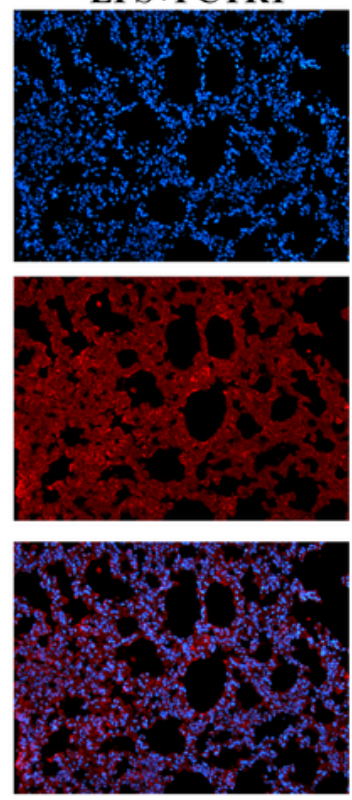

LPS+PCTR1
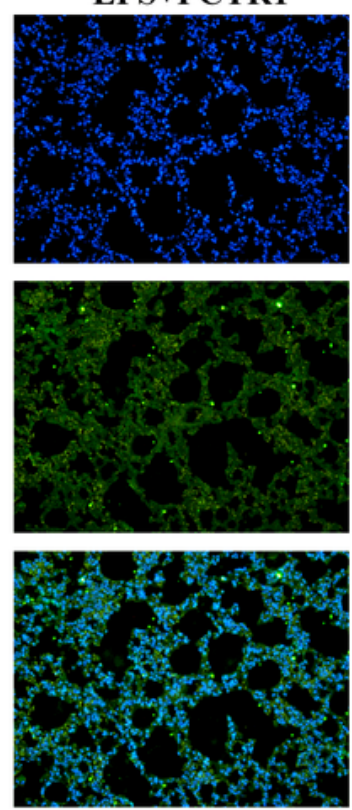

B

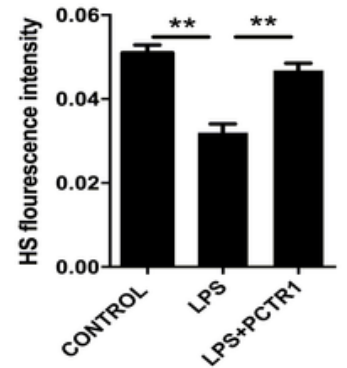

D

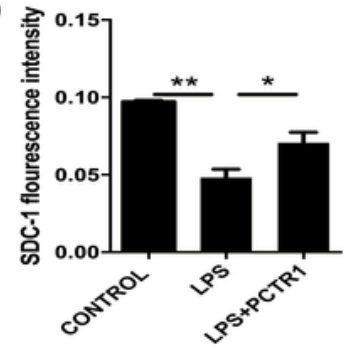

$\mathbf{E}$

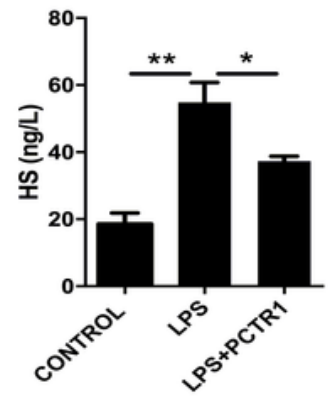

$\mathbf{F}$

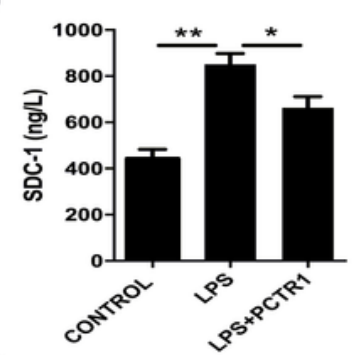

G

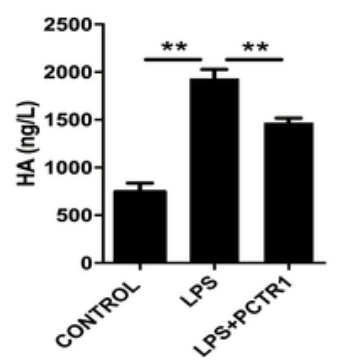

\section{Figure 3}

PCTR1 restores LPS-triggered endothelial glycocalyx damage in mice LPS $(10 \mathrm{mg} / \mathrm{kg})$ with or without PCTR1 (100 ng/mouse) was injected to mice for 6 hours. Representative image and densitometry of immunofluorescence of HS (a, b) and SDC-1 (c, d) in the lungs. Scar bar: $50 \mu \mathrm{m}$. (e-g) After collecting 
blood from the eyeballs, the serum levels of HS, SDC-1 and HA were quantified by ELISA. ${ }^{*} \mathrm{P}<.05$, ${ }^{*} \mathrm{P}<$ $.01, \mathrm{n}=5$.

\section{Figure 4}

A

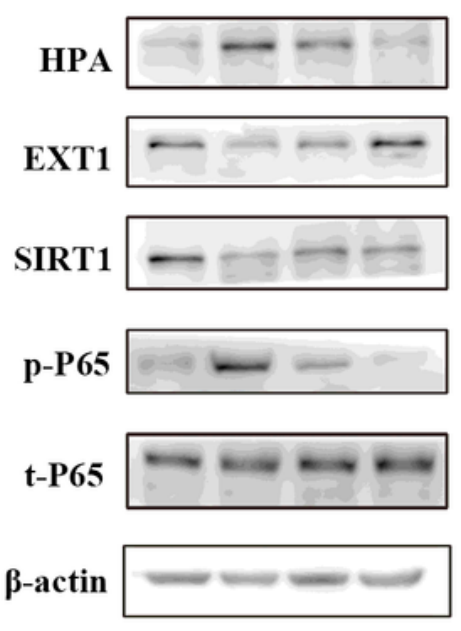

$62 \mathrm{kDa}$

86kDa

$110 \mathrm{kDa}$

$65 \mathrm{kDa}$

$60 \mathrm{kDa}$

43kDa
B

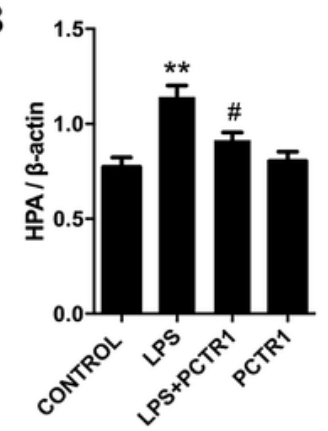

D

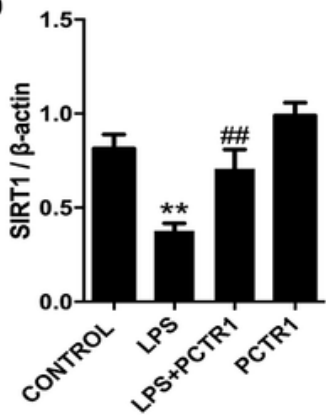

C

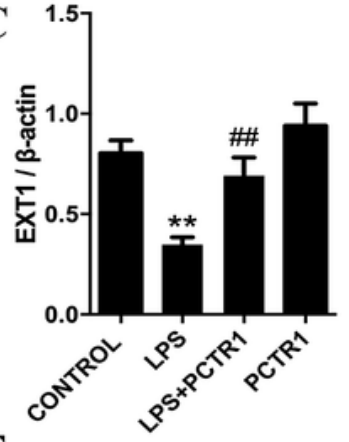

E
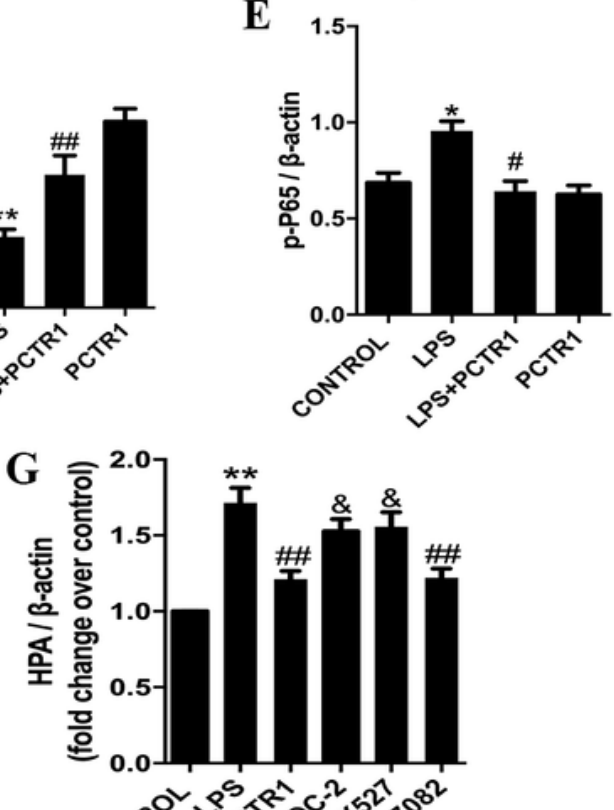

43kDa

62kDa

\section{EXT1}

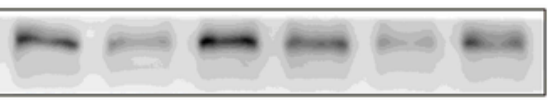

$\beta$-actin

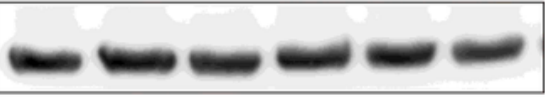

$\begin{array}{rcccccc}\text { LPS } & - & + & + & + & + & + \\ \text { PCTR1 } & - & - & + & + & + & - \\ \text { BOC-2 } & - & - & - & + & - & - \\ \text { EX527 } & - & - & - & - & + & - \\ \text { BAY11 } & - & - & - & - & - & +\end{array}$

\section{6kDa}
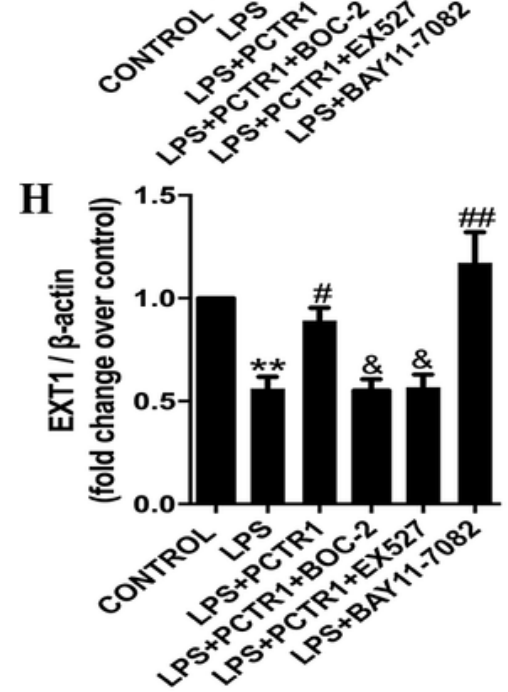

Figure 4

PCTR1 regulates HPA and EXT-1 expression in LPS- triggered sepsis in mice (a-e) Mice were treated with LPS (10 mg/kg) with or without PCTR1 (100 ng/mouse) for 6 hours. The levels of HPA, EXT-1, SIRT1 and p65 in the lung tissue were quantified by western blot. (f-h) BOC-2 (ALX receptor inhibitor, $600 \mathrm{ng} / \mathrm{kg}$ ) or 
EX527 (SIRT1 inhibitor冈10 mg/kg) or BAY11-7082 (NF-kB inhibitor , 20 mg/kg) was injected 1 hour followed by LPS $(10 \mathrm{mg} / \mathrm{kg}$ ) with or without PCTR1 (100 ng/mouse). The protein levels of HPA and EXT-1 were measured by western blot. ${ }^{*} P<.05$, $* * P<.01$ relative to the CONTROL group, \# $P<.05$, \#\# $P<.01$ relative to the LPS group $₫$ \&P $<.05$, relative to the LPS+PCTR1 group. $n=6$.

FIGURE 5

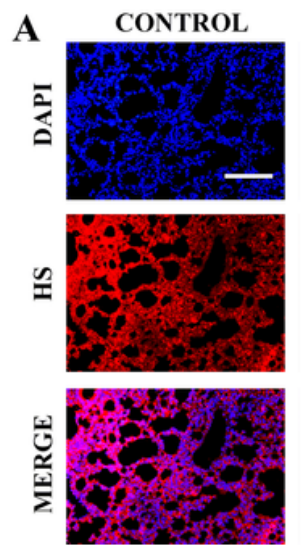

LPS
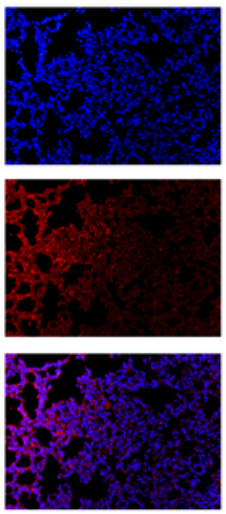

C CONTROL
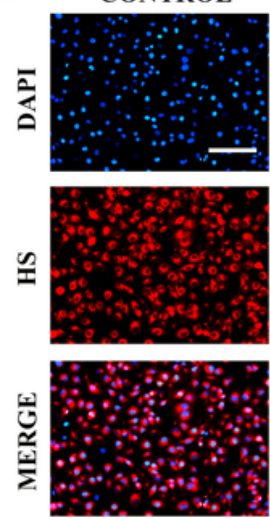

LPS+PCTR1
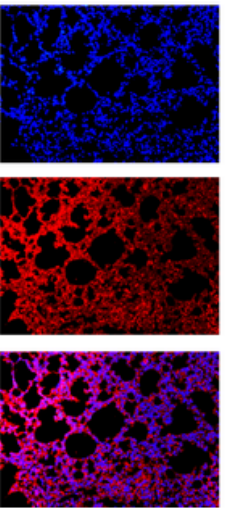

LPS+PCTR
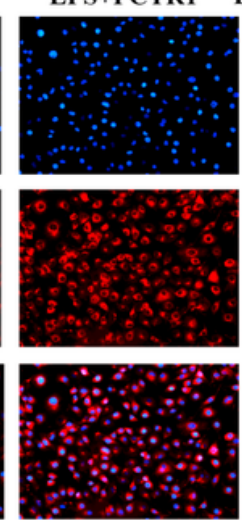
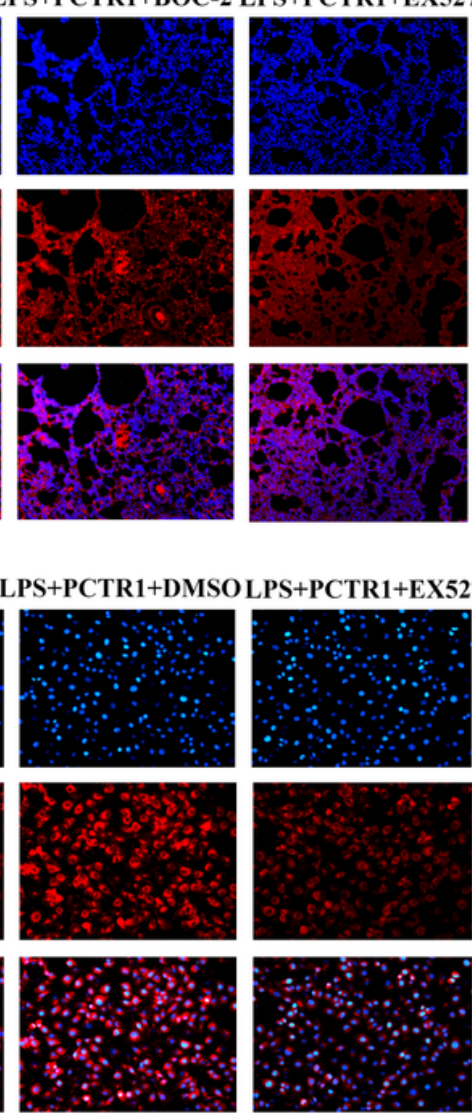
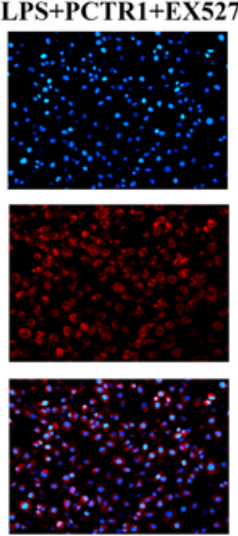
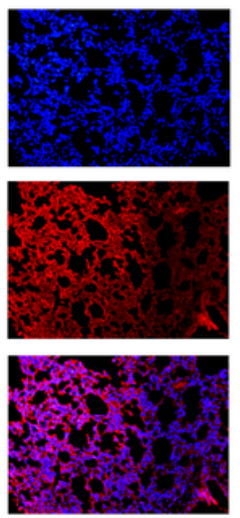

B
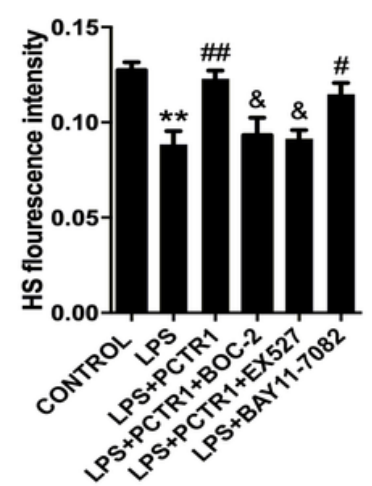

$\mathbf{D}$
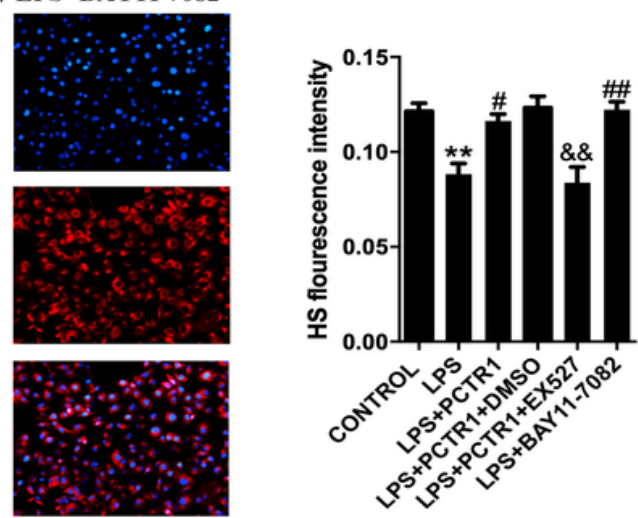

Figure 5

PCTR1 protects the endothelial glycocalyx by modulating SIRT1/NF-KB pathways via ALX receptor The level of HPA, EXT-1, SIRT1 and p65 in lung tissues was quantified using western blotting assay. BAY117082 (NF-KB inhibitor , 20 mg/kg) or EX527 (SIRT1 inhibitor冈10 mg/kg) or BOC-2(ALX receptor inhibitor, $600 \mathrm{ng} / \mathrm{kg}$ ) was injected 1 hour followed by LPS (10 mg/kg) with or without PCTR1 (100 ng/mouse) for 6 hours. $(a, b)$ The expression of HS in tissue was examined by immunofluorescence. HUVECs were administered with $1 \mu \mathrm{g} / \mathrm{ml}$ LPS and $100 \mathrm{nM}$ PCTR1 for 6 hours. After treatment with EX527 or DMSO, HS in HUVECs was determined by immunofluorescence $(c, d)$. Scar bar $=50 \mu \mathrm{m}$. **P $<.01$ in comparison to the CONTROL group, \# P $<.05$, \#\# $\mathrm{P}<.01$ in comparison to the LPS group $\& \mathrm{P}<.05 \rrbracket \& \& P<.01$ \in comparison to the LPS+PCTR1 group. $n=6$. 


\section{Figure 6}

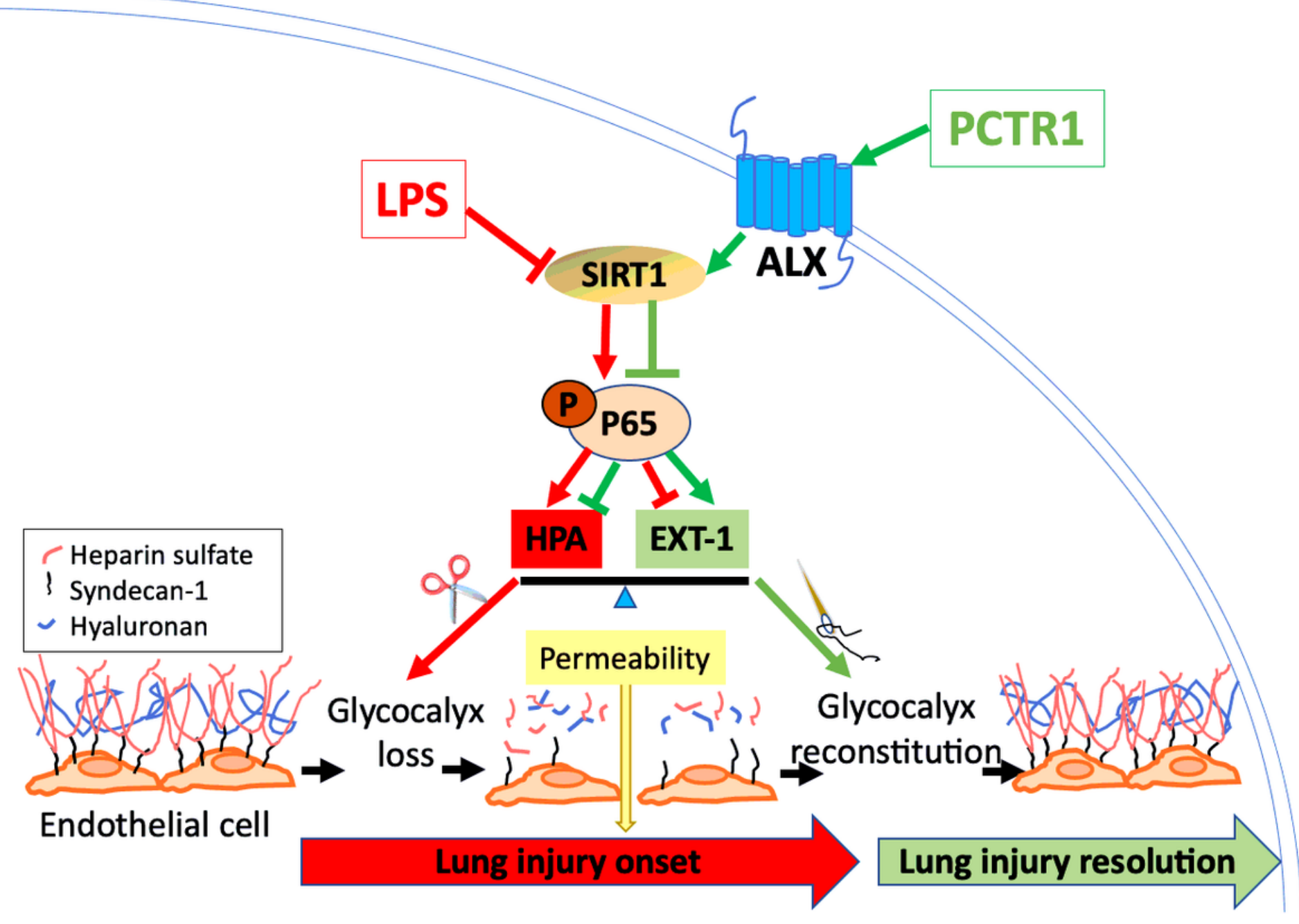

Figure 6

PCTR1 restores Lipopolysaccharide-induced pulmonary endothelial glycocalyx via ALX/SIRT1/NF-kappa $B$ axis in mice. The effect of LPS was shown in red, whilst the effect of PCTR1 was shown in green. ALX: a G protein-coupled receptor; LPS: Lipopolysaccharides; PCTR1: Protectin conjugates in tissue regeneration 1; SIRT1: sirtuin 1; HPA: heparanase; EXT-1: exostosin-1. 